\title{
Magnetic Interfaces at the Nanoscale: From Fundamentals to Technological Applications
}

\author{
M. Angelakeris, ${ }^{1}$ O. Crisan, ${ }^{2}$ and C. Martinez-Boubeta ${ }^{3}$ \\ ${ }^{1}$ Department of Physics, Aristotle University of Thessaloniki, Thessaloniki, Greece \\ ${ }^{2}$ National Institute for Materials Physics, Bucharest, Romania \\ ${ }^{3}$ Department of Electronics, University of Barcelona, Barcelona, Spain
}

Correspondence should be addressed to M. Angelakeris, agelaker@auth.gr

Received 12 July 2012; Accepted 12 July 2012

Copyright (c) 2012 M. Angelakeris et al. This is an open access article distributed under the Creative Commons Attribution License, which permits unrestricted use, distribution, and reproduction in any medium, provided the original work is properly cited.

One of the most widely researched topics over the last decades has been magnetic nanoscale systems, but recent breakthroughs in high-resolution imaging techniques in modern computing and electronics towards continuously smaller devices have nourished a booming in the quest for new materials and their novel applications. It is unambiguous that the interest for tailor-made and properly functionalized particles is very wide and expected results will be of enormous benefit for the global economy. Still, researchers should improve the manufacture of nanomaterials on a larger scale at a lower cost. In nanotechnology, methods to produce some desired structure must satisfy certain conditions, such as speed, performance, and precision. Although computer modelling may provide a hint in finding a structure that minimizes the internal energy, control of nanoscale phenomena in real time remains a puzzle in most cases. In this regard, even if certain prerequisites should be fulfilled for specific applications, magnetic nanoscale systems exploitation lays in the control of their interface properties.

Nanomagnets may enter in the production of almost every product we use, as standard magnets do. Among them, magnetic nanoparticles deserve special attention because of their extended applicability not only for data storage and nanoscale spintronics applications but also for healthcare. Medical researchers are actively seeking to develop improved tools for diagnosis (gene sequencing, high contrast agents for imaging) and new therapies such as hyperthermia where functionalized magnetic nanoparticles may target only the tumors without harming the healthy tissues or could be used in synergy with chemotherapeutic drug delivery.

This special issue provides a carefully selected compilation of original research papers as well as review articles in understanding and insight of magnetic properties at nanoscale interfaces. Our scientific goal was to collect articles focusing on chemical and magnetic interfaces of nanostructured materials, in determining their influences on intrinsic magnetic properties, and to trace the role of these issues in the macroscopic behavior of nanostructures.

We did our best to conduct review and editing processes according to international standards, and we believe this special issue addresses a novel emerging technology, namely the control, functionalization, and analysis of nanometersized magnetic materials. Eventually, it turned out that about $50 \%$ of submitted papers have been finally accepted for publication. Finally, we would like to thank all the reviewers for their prompt and substantial involvement and all the authors for the interesting and fruitful results presented in this issue and we hope that especially young scientists will benefit from this multidisciplinary special issue, which is relevant to various up-to-date and future nanotechnologies.

M. Angelakeris O. Crisan C. Martinez-Boubeta 

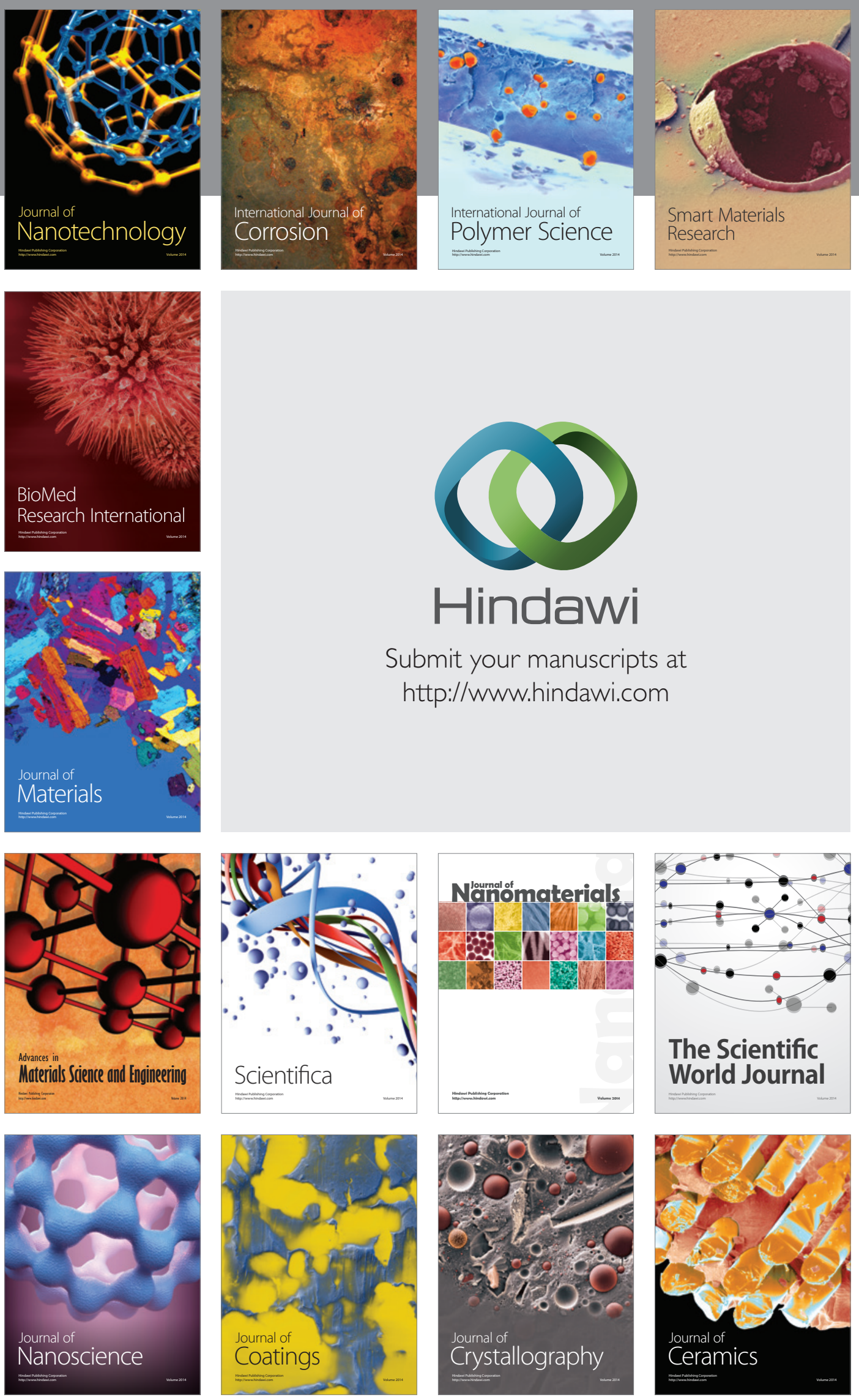

The Scientific World Journal

Submit your manuscripts at

http://www.hindawi.com

\section{World Journal}

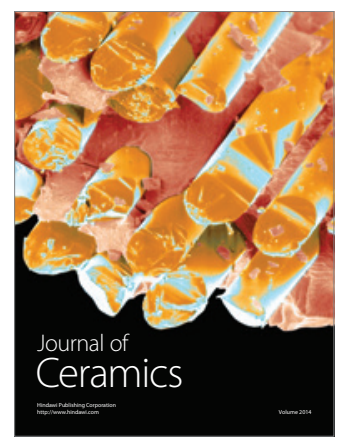

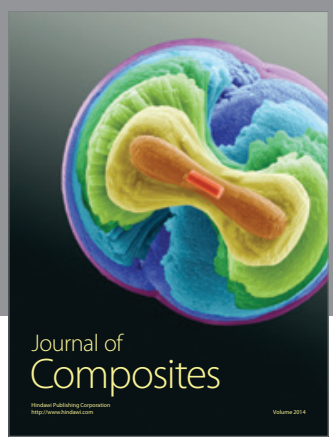
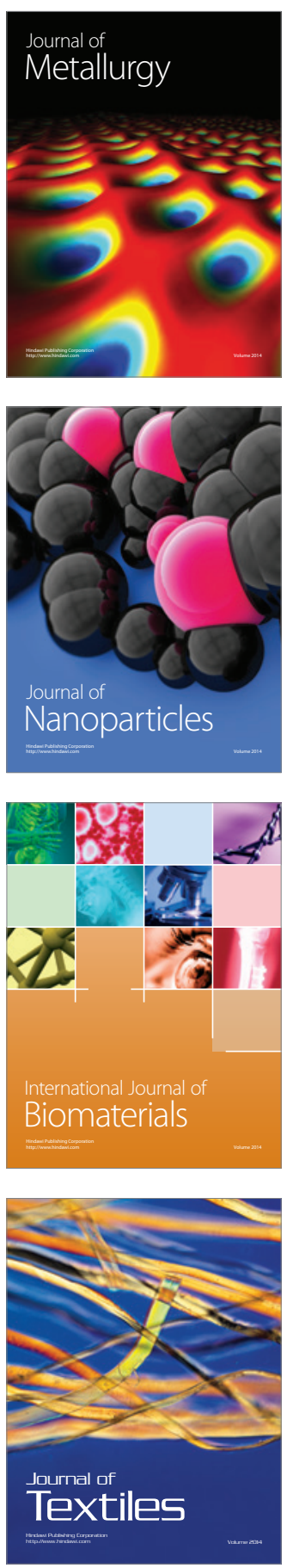